\title{
Temporal pattern of cysteine endopeptidase (cathepsin B) expression in cartilage and synovium from rabbit knees with experimental osteoarthritis: gene expression in chondrocytes in response to interleukin-1 and matrix depletion
}

\author{
Fuad Mehraban, Michael H Tindal, Michelle M Proffitt, Roland W Moskowitz
}

\begin{abstract}
Objective-To determine the temporal pattern of expression of cathepsin-B in chondrocytes and synovium in experimental osteoarthritis, and to determine possible mechanisms for upregulation and secretion of cathepsin-B from chondrocytes.

Methods-Experimental osteoarthritis was induced with partial medial meniscectomy (PM); sham operated (SH) and normal $(\mathrm{N})$ rabbits were used as controls. Cathepsin-B mRNA expression was assessed with northern blotting with $a{ }^{32} \mathbf{P}$ labelled cDNA probe. Cathepsin-B was measured in conditioned media or cell extracts using a fluorogenic substrate Z-Arg-Arg-AMC. Chondrocyte monolayers were used to determine cathepsin-B expression in response to interleukin-1 $\beta$ (IL-1ß). Cartilage explants were used to test the effect of matrix depletion on cathepsin-B release.
\end{abstract}

Results-Chondrocytes obtained from experimental osteoarthritis knees did not show cathepsin-B mRNA upregulation. However, isolated chondrocytes secreted cathepsin-B into the culture medium. Enzyme release was significantly higher at 8 weeks relative to controls, but not at 12 weeks or 4 weeks. Enzyme released from synovium was significantly higher in PM group compared with SH group at 4 and 8 weeks. IL-1 $\beta$ was ineffective in upregulating steady state cathepsin-B mRNA in chondrocytes; however, it upregulated the intracellular enzyme, and this was blocked with cycloheximide. Enzymatic depletion of cartilage matrix after exposure of explants to IL-1 resulted in release of significantly higher amounts of cathepsin-B into the medium by matrix depleted chondrocytes compared with intact explants.

Conclusions-In experimental osteoarthritis, cathepsin-B is upregulated in synovial tissue during the early degenerative phase. Progression of experimental osteoarthritis is accompanied by upregulation of cathepsin-B in cartilage. Cartilage and synovial cathepsin-B levels decline as experimental osteoarthritis advances to more degenerative states. IL-1 upregulates intracellular cathepsin-B by increasing cathepsin-B protein synthesis; it is not an effective stimulus for enzyme secretion. Depletion of cartilage matrix during progression of experimental osteoarthritis may contribute to secretion of cathepsin-B and perpetuation of cartilage destruction.

(Ann Rheum Dis 1997;56:108-115)

The degradation of cartilage in osteoarthritis is thought to be due to the action of a number of proteases synthesised and secreted by cartilage and synovial tissue in response to noxious stimuli. Well known among these are the neutral matrix metalloproteases (MMP) thought to be active in disease pathogenesis. ${ }^{1}$ Other enzymes have also been shown to be capable of degrading extracellular matrix macromolecules. Cathepsin-B, a lysosomal cysteine proteinase, can cleave aggrecan at a site near to that of MMP-3 (stromelysin). ${ }^{2}$ It is also active against fibronectin at both acid and neutral $\mathrm{pH},{ }^{3}$ and can activate pro-stromelysin in vitro. ${ }^{4}$ Both cathepsin-B and cathepsin-L were found to degrade collagen types II, IX, and XI in the non-helical domains, ${ }^{5}$ and cartilage link protein as well. ${ }^{6}$ The finding of increased cathepsin-B in cartilage and synovium of osteoarthritic patients lends further credence for cathepsin-B involvement in the pathogenesis of osteoarthritis. ${ }^{7}$ Recent reports on the histochemical and pathological distribution of cathepsin-B in human osteoarthritic cartilage suggested that it may be involved in the perpetuation of osteoarthritis rather than in the initial assault on cartilage. ${ }^{89}$

Of note, it has been shown that a specific inhibitor of cathepsin-B, N-(L-3-trans-propylcarbomoyloxirane-2-carbonyl)-L-isoleucyle-Lproline (CA074) and its lipophilic methyl ester, when tested at nanomolar concentrations in a tissue culture system of bovine chondrocytes, inhibited the proteoglycan release brought about by interleukin-1 (IL-1) treatment, but could not inhibit the retinoic acid stimulated proteoglycan fragments released from these chondrocytes. ${ }^{10}$ Lipophilicity of the compound was necessary for this effect, suggesting an intracellular site of action. Fluoroketone cysteine proteinase inhibitors were found to decrease articular cartilage and 
bone loss associated with chronic inflammatory arthritis model. ${ }^{11}$ It is possible that cathepsin-B is involved in degradation of extracellular matrix, either by taking part in activation of matrix metalloproteases such as stromelysin, or alternatively by a direct action on matrix components. This would make proteoglycans more easily accessible to degradation by other proteases in the chain of catabolism.

Stimulation of rabbit chondrocytes in monolayer culture with IL-1 resulted in increased accumulation of cathepsin-B in intracellular granules but no significant release of enzyme activity. ${ }^{12}$ In another study, stimulation of synovial fibroblast-like cells from rheumatoid arthritis patients with IL-1 or tumour necrosis factor- $\alpha$ led to increased secretion of cysteine proteinase activity which appeared to represent primarily cathepsin-B. ${ }^{13}$ Of interest, it was shown that IL-1-stimulated proteoglycan release from bovine nasal septum explants was mediated at least in part through cysteine proteinases. ${ }^{14}$ A recent study showed that cathepsin-B was increased in and around zones of active cartilage destruction in osteoarthritis. ${ }^{8}$ The stimulus for the release of cathepsin-B (an intracellularly stored endopeptidase) in cartilage has not been addressed.

In this study we evaluated the expression of cathepsin-B in experimental osteoarthritis in chondrocytes and synovium at different time points corresponding to early degenerative lesions [four weeks after partial medial meniscectomy (PM)], and progressive stages of experimental osteoarthritis (8 and 12 week post-PM). We also examined possible mechanisms whereby cathepsin-B may be regulated and released from osteoarthritic tissue. The significance of these findings is discussed in relation to a postulated role of cathepsin-B in osteoarthritis.

\section{Methods}

Experimental osteoarthritis (EOA) was induced in both knees of female New Zealand white rabbits (specific pathogen-free, six to eight weeks old, 1.8-2.3 kg; Hazelton Laboratories, Denver, PA, USA) using the technique of partial medial meniscectomy (PM) as described. ${ }^{15}$ Sham operated $(\mathrm{SH})$ and non-operated $(\mathrm{N})$ rabbits served as controls. Eighty seven rabbits were used in the study over a period of two years $(n=29$ for each group designated $\mathrm{N}, \mathrm{SH}, \mathrm{PM}$ ). These were divided up and killed at intervals of four weeks ( $n=33 ; 11$ in each group), 8 weeks $(n=33,11$ in each group), and 12 weeks ( $n=21$, seven in each group) postsurgery. Joint pathology was recorded at the time the animals were killed.

\section{TISSUE CULTURE METHODS}

Reagents and media

All enzymes used for cell dissociation were from Worthington Biochemical Corporation, Freehold, NJ, USA; tissue culture media and serum were obtained from Gibco BRL, Grand Island, NY.
Suspension cultures-Cartilage was shaved under sterile conditions and pooled from both legs of each rabbit. Chondrocytes were liberated from matrix by sequential enzymic digestion as follows. Enzymes were dissolved at the indicated concentrations in Gey's solution and sterilised by passage through a 0.45 micron filter. Cartilage pieces were softened by treatment for five minutes with $0.5 \mathrm{mg} \mathrm{ml}^{-1}$ hyaluronidase (Bovine testes). Cartilage was then cut up further into smaller pieces and digested with $2 \mathrm{mg} \mathrm{m}^{-1}$ trypsin (Bovine pancreas) for half an hour at $37^{\circ} \mathrm{C}$, followed by $2 \mathrm{mg} \mathrm{m}^{-1}$ collagenase (type 2 from Clostridium histolyticum) for one and half to two hours at $37^{\circ} \mathrm{C}$. One million cells were cultured at a density of $2 \times 10^{5}$ cells $\mathrm{ml}^{-1}$ for three days in Ham's F12 medium containing $0.2 \%$ lactalbumin hydrolysate, penicillin, streptomycin, and Fungizone $(\mathrm{F} 12 / \mathrm{LAH})$. Under these conditions cells remained as a suspension culture with no cell division. Viability was $95 \%$ or more at the end of the culture (three days). The conditioned medium was filtered through 0.45 $\mu$ filters and frozen at $-20^{\circ} \mathrm{C}$ until used. Enzyme assays were performed within 24 hours.

Monolayers-For monolayer chondrocytes, cartilage was pooled from knees, shoulders, and hips. Chondrocytes obtained after digestion as above were plated at an initial density of $2 \times 10^{5} \mathrm{ml}^{-1}$ in Ham's F12 medium supplemented with $10 \%$ heat inactivated bovine fetal serum and antibiotics (F12/ FBS) as noted above. Medium was changed every three days until the monolayers were confluent.

Cartilage explants-Cartilage from knees, shoulders, and hips was shaved, pooled, and cut into small pieces (approximately $2 \mathrm{~mm} \times 2$ $\mathrm{mm})$. These were weighed and $0.2 \mathrm{~g}$ was put into each well of a multi-well plate (Falcon) with $4 \mathrm{ml}$ of Dulbecco's Modified Eagle's medium (DMEM) containing antibiotics and $0.2 \%$ LAH (DMEM/LAH).

Synovial cultures-Synovium was dissected away from the subjacent patellar ligament and those from both legs of each rabbit were pooled. The synovia were cut into small pieces and cultured as above for three days. Conditioned medium was collected, passed through a 0.45 micron filtered, and stored at $-20^{\circ} \mathrm{C}$ until used. Assays were performed within 24 hours.

PREPARATION OF TOTAL CELLULAR RNA

Freshly prepared chondrocytes were used for preparation of total cellular RNA by Tri Reagent method (Molecular Research Center, Cincinnati, Ohio, USA) using the manufacturer's recommended method.

\section{MEASUREMENT OF CATHEPSIN-B ACTIVITY}

Cathepsin-B activity was measured in the medium as described using a fluorogenic substrate Z-Arg-Arg-AMC ${ }^{14}$ (Bachem Bioscience, King of Prussia, PA, USA). We modified the method for culture medium as follows. Culture medium to be assayed was acidified with $1 \mathrm{M} \mathrm{HCl}$ to $\mathrm{pH} 5.0$. A $200 \mu \mathrm{l}$ aliquot of sample was added to $1.8 \mathrm{ml}$ of assay buffer 
containing $50 \mathrm{mM}$ of [2-N-morpholinoethanesulphonic acid (Mes), $4 \mathrm{mM}$ cysteine, $0.01 \%$ Brij-35, $2 \mathrm{mM}$ EDTA], and $5 \mu \mathrm{M}$ Z-Arg-Arg-AMC, $\mathrm{pH}$ 5.5. The mixture was incubated for 18 hours at $37^{\circ} \mathrm{C}$ and the reaction products quantitated by determination of relative fluorescence with excitation at $360 \mathrm{~nm}$ and emission at $460 \mathrm{~nm}$. The fluorescence was standardised with a known amount of aminomethylcoumarine (AMC) (Sigma, St Louis, MO, USA) in the same buffer. Number of moles of substrate hydrolysed was calculated. One unit of enzyme activity is defined as that releasing $1 \mu \mathrm{mol} \mathrm{min}{ }^{-1}$ of AMC from substrate. For measurement of total enzyme activity (latent + active) the latent enzyme was first activated by treating the acidified medium with $10 \mu \mathrm{g} \mathrm{ml}^{-1}$ of pepsin for 30 minutes at $37^{\circ} \mathrm{C}$. Blank or background fluorescence was determined with substrate alone in medium, or by addition of $5 \mu \mathrm{M}$ E64 (Sigma) to samples.

PREPARATION OF CDNA PROBE FOR RABBIT CATHEPSIN-B

Rabbit liver poly A+ RNA was reverse transcribed into first strand cDNA using Superscript reverse transcriptase (BRL, Grand Island, NY, USA). A 500 base pair cDNA fragment of the rabbit cathepsin-B was obtained using the polymerase chain reaction (PCR) by amplification of DNA sequences flanked by two degenerate oligonucleotide primers designed by consideration of the known human, mouse, and rat cDNA sequences. ${ }^{16}$ The primers used were as follows. A $42 \mathrm{mer}$ sense primer 5' GTCAAGCTTTGTGGGGA (CT) GGCTGTAATGGTGGCTATCC 3') encompassing nucleotides $436-464$ plus extra nucleotides forming a Hind restriction site at the $5^{\prime}$ end, and a 48 mer anti-sense primer ( $5^{\prime}$ ATGAAGCTTTCCTCTGAG(TG)ATTTTAAAGA A(GA)C CATT(AG) TCACCCCA encompassing nucleotides 910-945 plus extra sequences forming a Hind III restriction site at the $3^{\prime}$ end. The resultant cDNA was inserted into pBluescript II KS phagemid (Stratagene, La Jola, CA, USA) through the incorporated Hind III sites and cloned into $E$ coli. After plating on ampicillin agar plates containing $\mathrm{X}$-gal and isopropyl thiogalactoside, colonies were screened by digestion of plasmid DNA with Hind III enzyme. Several clones with the right insert size $(500 \mathrm{bp})$ were sequenced using the dideoxynucleotide chain termination sequencing method $^{17}$ using M13 forward and reverse primers. Of these, three were found to have a high degree of homology with the published sequences of human, mouse, and rat cathepsin-B cDNAs. One clone was chosen for preparation of cDNA used in this study.

NORTHERN BLOTTING

The cathepsin-B cDNA was labelled with ${ }^{32} \mathrm{P}-\alpha$-deoxycytidine $\left({ }^{32} \mathrm{P}-\alpha-\mathrm{dCTP}\right)$ triphosphate using random primed reaction (Boehringer Mannheim, Indianapolis, IN, USA), and was used to probe northern blots as described. ${ }^{18}$ To ascertain that chondrocytes were responsive to IL-1, a rabbit pro-stromelysin cDNA probe was used to reprobe some of the blots. The characterisation of the pro-stromelysin cDNA has been described in detail previously. ${ }^{18}$ After probing with cathepsin-B cDNA probe and exposure to $x$ ray film, the first probe was stripped by boiling the blots in RNase-free water. Blots were reprobed with a control ${ }^{32} \mathrm{P}$-cDNA probe for polypeptide elongation factor-1 $\alpha$ (PEF) (obtained from Thomas Hering $\mathrm{PhD}$, Case Western Reserve University, Cleveland, $\mathrm{OH}, \mathrm{USA}$ ) and exposed to obtain a control for variations in gel loading and blot transfer. Plasmid containing GAPD cDNA insert was obtained from American Type Culture Collection (Rockville, MD, USA).

NORMALISATION OF CATHEPSIN-B MRNA BAND INTENSITIES

To obtain a relative measure of cathepsin- $B$ mRNA expression in $\mathrm{N}, \mathrm{SH}$, and $\mathrm{PM}$ chondrocytes, cathepsin-B mRNA band intensities were normalised to the corresponding PEF mRNA band intensities. This was achieved by evaluation of the autoradiographic band intensities by densitometry. Relative expression was calculated as the ratio of the intensity of cathepsin-B mRNA band to that of the corresponding PEF band.

\section{CATHEPSIN-B STEADY STATE MRNA LEVEL IN} RESPONSE TO IL-1 $\beta$

Confluent rabbit chondrocyte monolayers were taken into LAH medium for 24 hours. Varying doses of IL- $1 \beta$ were added to cultures, followed by incubation at $37^{\circ} \mathrm{C}$ for 24 hours. At intervals total cellular RNA was made from the cell layer and a northern blot prepared. This was probed sequentially with ${ }^{32} \mathrm{P}$-labelled cathepsin-B cDNA, stromelysin cDNA, and control cDNA probes PEF and GAPD.

EFFECT OF IL-1 ON INTRACELLULAR AND SECRETED CATHEPSIN-B, AND ITS BLOCKADE WITH CYCLOHEXIMIDE

Confluent rabbit chondrocyte monolayers were taken into LAH medium for 24 hours. Varying doses of IL-1 $\beta$ with or without $10 \mu \mathrm{M}$ cycloheximide was added to cultures, followed by incubation at $37^{\circ} \mathrm{C}$ for 24 hours. Cathepsin-B activity was measured in cell layer and culture medium.

EXTRACTION OF CATHEPSIN-B FROM CHONDROCYTE MONOLAYERS

Monolayers were washed with Gey's solution, scraped with a cell lifter, and centrifuged to pellet the cells. The cell pellet was homogenised in $50 \mathrm{mM}$ Mes buffer, $\mathrm{pH}$ 5.5, containing $0.01 \%$ Brij-35, 2 mM EDTA, and 4 $\mathrm{mM}$ cysteine. The homogenate was frozen for 30 minutes at $-20^{\circ} \mathrm{C}$, thawed, and centrifuged for 15 minutes at $8000 \mathrm{~g}$ to pellet cell debris; the supernatant was taken for assay.

CATHEPSIN-B SECRETION IN RESPONSE TO MATRIX DEPLETION

Explants were cultured for two days in DMEM/LAH medium, the medium was changed, and IL- $1 \beta$ was added. After further two days medium was collected and frozen at $-20^{\circ} \mathrm{C}$ until assayed. Chondrocytes were 

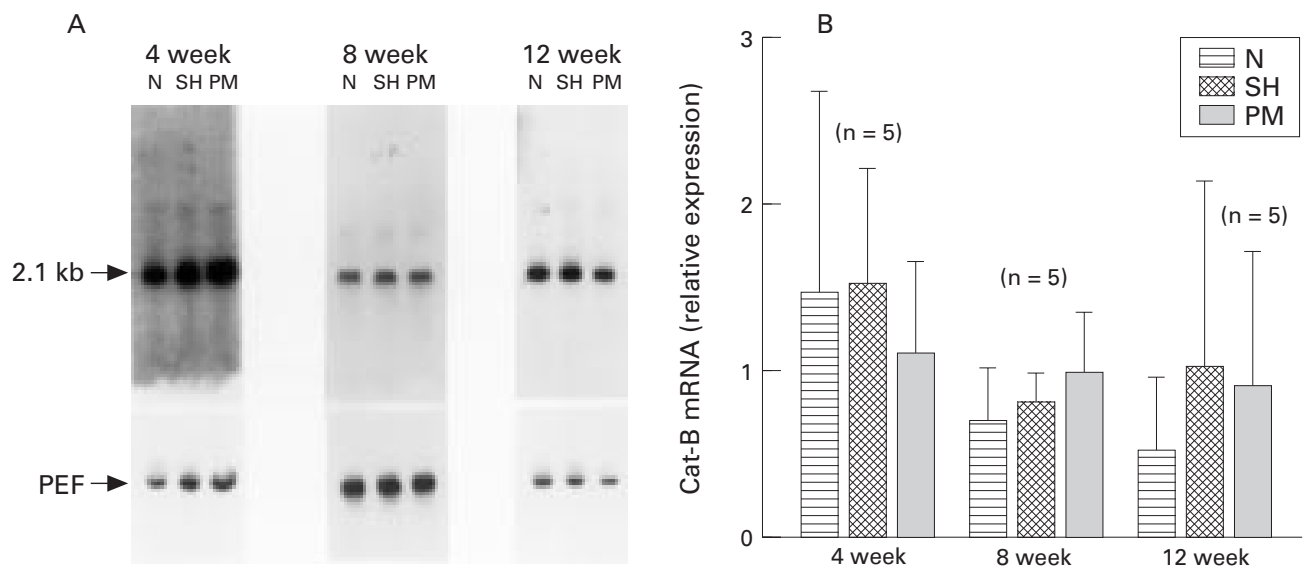

Figure 1 (A) Representative northern blot exposures of total cellular RNA from $N$ (non-operated control), SH (sham operated), and PM (partial meniscectomy) cartilage at 4, 8, and 12 weeks after surgery. Blots were probed with a ${ }^{32} P$ labelled cDNA probe for rabbit cathepsin-B. After exposure the blots were reprobed with control cDNA probe polypeptide elongation factor-1 (PEF) for normalisation of band intensities. (B) Northern blots as in $A$ were scanned and band intensities determined. The intensities of cathepsin- $B \mathrm{mRNA}$ bands were normalised to that of the corresponding PEF $m R N A$ bands. Relative expression was calculated as the ratio of cathepsin- $B m R N A$ band intensity to that of PEF The average (error bar $=S D$ ) of five separate northern blots in each case is shown. The relative expression of PM group was not significantly different to that of $\mathrm{N}$ and $S H$ groups at any time point.

obtained by digestion of matrix with collagenase and the cells were placed into suspension culture in F12/LAH medium; after two days the medium was separated from cells and assayed.

OTHER REAGENTS

Recombinant human IL-1 $\beta$ (rh IL-1 $\beta$ ) was a gift from Dr Stephen Eisenberg and Dr James L Vannice (Synergen, Boulder, CO, USA). Other chemicals were molecular biology grade obtained from BRL or Sigma. For radiolabelling, ${ }^{32} \mathrm{P}-\alpha-\mathrm{dCTP}$ (specific activity $3000 \mathrm{Ci} \mathrm{mmol}^{-1}$ ) was obtained from New England Nuclear (Boston, MA, USA).

STATISTICAL ANALYSES

The significance of the data was determined by comparison of the mean of experimental groups using Student's two tailed $t$ test with significance at $\alpha$ level of 0.05 . Statistical parameters of kurtosis and skewness were calculated. The data were normally distributed if the numerical values of kurtosis and skewness

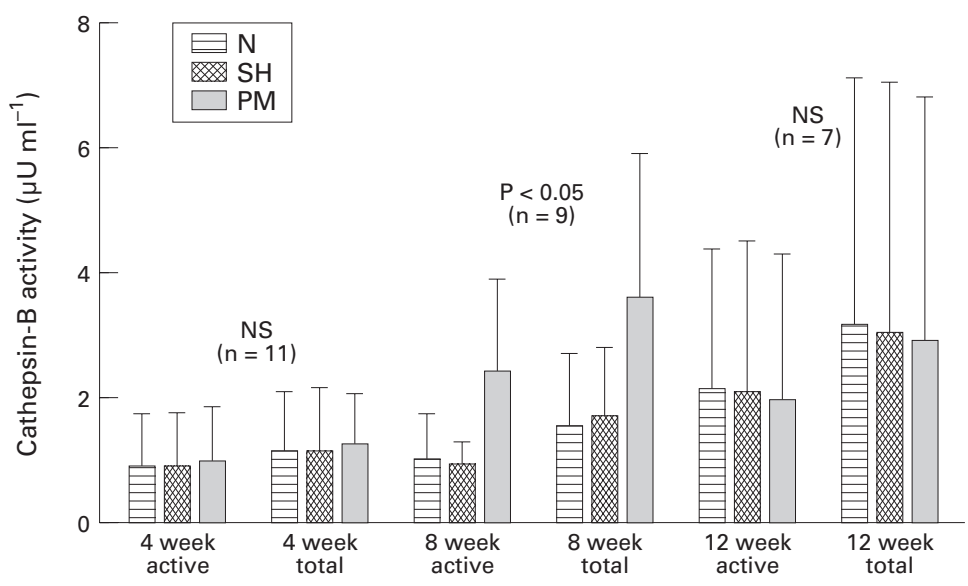

Figure 2 Cathepsin-B activity (active and total) was measured in chondrocyte conditioned media (CCM) from $N$ (non-operated control), SH (sham operated), and PM (partial meniscectomy) rabbits. Average values (error bars $=S D$ ) are shown; $n$ is the number of rabbits in each experimental group $(N, S H, P M) . N S=$ not statistically significant. were between -2 and +2 . A $\chi^{2}$ test was used to determine the statistical significance between the means of paired points comprising dose-response curves in culture experiments.

\section{Results}

CHARACTERISTICS OF CATHEPSIN-B CDNA

Analysis of the cloned rabbit cathepsin-B cDNA sequence showed a strong homology with the published sequences of human, mouse, and rat cDNAs. ${ }^{16}$ There were 28 nucleotide substitutions unique for rabbit cathepsin-B in the 507 bp cloned cDNA (overall $95 \%$ homology compared with human). On northern blots of rabbit chondrocyte total cellular RNA (cRNA) this cDNA hybridised with a $2 \mathrm{~kb}$ mRNA, the expected size of cathepsin-B mRNA. A minor band of approximately $4.0 \mathrm{~kb}$ was also hybridised with this probe. This minor mRNA species probably represents an alternatively spliced mRNA also seen in human tissue. ${ }^{19}$

\section{STEADY STATE CATHEPSIN-B MRNA IN EOA}

CHONDROCYTES

Figure $1 \mathrm{~A}$ is a representative northern blot of cRNA from knee chondrocytes of $\mathrm{N}, \mathrm{SH}$, and PM rabbits obtained at 4,8 , and 12 weeks after surgery. There was no discernible difference in the level of expression of steady state mRNA for cathepsin-B in EOA chondrocytes versus $\mathrm{N}$ and $\mathrm{SH}$ chondrocytes at time points representing early ( 4 weeks) and increasingly progressive stages of experimental osteoarthritis (8 and 12 weeks post-PM). The average intensities of cathepsin-B mRNA bands were normalised to that of control probe, PEF, by densitometric measurements performed on similar blots from five rabbits in each group $(\mathrm{N}, \mathrm{SH}, \mathrm{PM})$ at 4,8 , and 12 weeks. Figure $1 \mathrm{~B}$ shows the mean of the relative expression (error bars $=\mathrm{SD}$ ) of cathepsin-B mRNA bands obtained for the groups. No statistically significant differences were found in mRNA band intensities (at any given time) of osteoarthritic rabbits compared with the controls. 


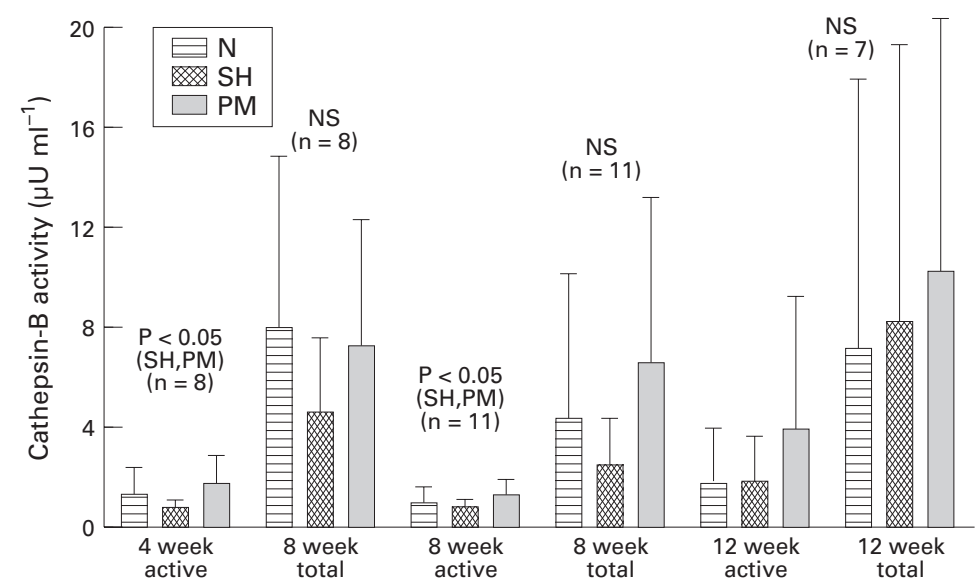

Figure 3 Cathepsin-B activity (active and total) was measured in synovial conditioned media (SCM) from $N$ (non-operated control), SH (sham operated), and PM (partial meniscectomy) rabbits. Averages (error bars $=S D$ ) are shown; $n$ is the number of rabbits in each experimental group $(\mathrm{N}, S H, P M) . N S=$ not statistically significant.

CATHEPSIN-B ENZYME ACTIVITY UPREGULATION IN CHONDROCYTES AND SYNOVIUM OF EOA RABBITS.

When EOA chondrocytes were cultured in LAH medium they maintained a spherical morphology in a suspension culture with no cell division. Cell viability was $95 \%$ or more at the end of culture when medium was collected. There was no detectable lactate dehydrogenase

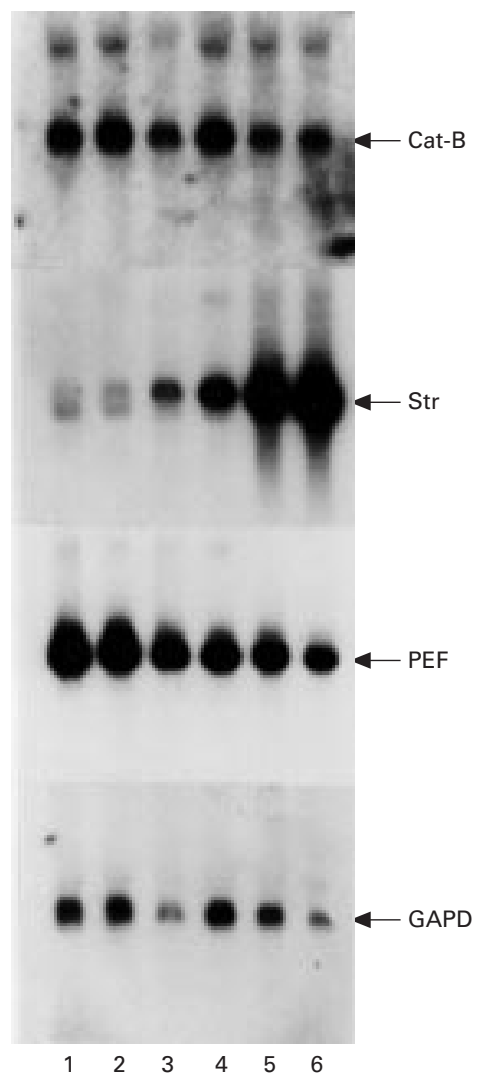

Figure 4 Northern blot exposures of total cellular RNA from monolayer chondrocytes probed sequentially with ${ }^{32} \mathrm{P}$ labelled cDNA probe for rabbit cathepsin-B (Cat-B), rabbit stromelysin (Str), polypeptide elongation factor-1a (PEF), and GAPD. Confluent monolayers of rabbit articular chondrocytes were transferred into serum-free medium for

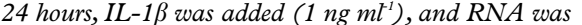
harvested at time intervals $0,1,2,4,8$, and 16 hours and loaded in lanes 1-6 respectively.

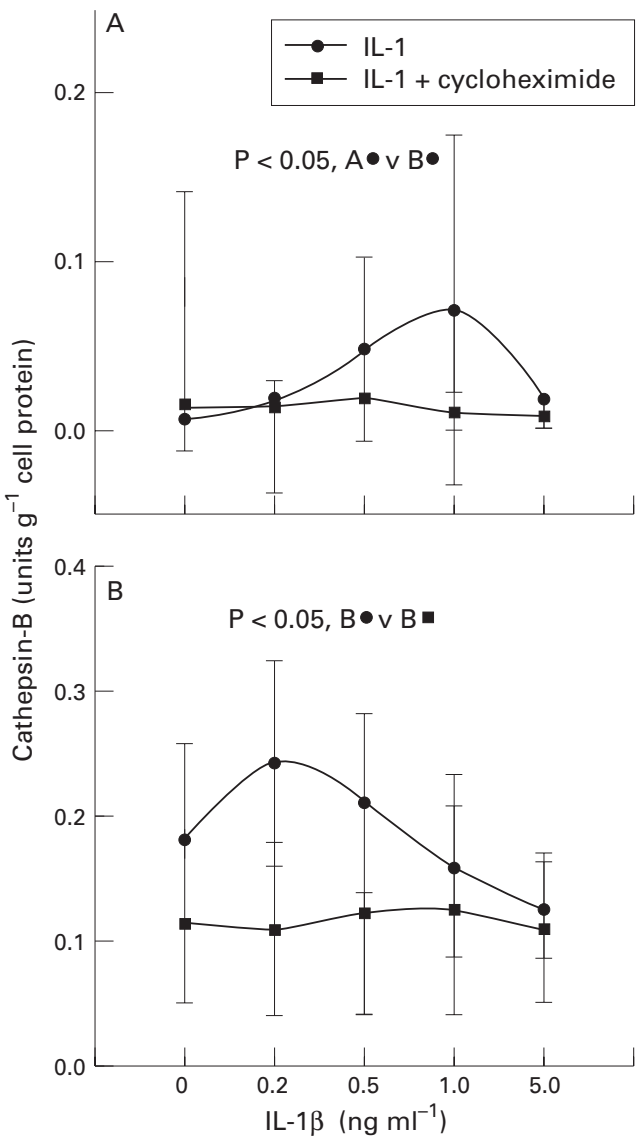

Figure 5 Confluent monolayer cultures of rabbit articular chondrocytes were transferred into serum-free medium for 24 hours. Various doses of $I L-1 \beta$ were added to cultures with or without the addition of $10 \mu \mathrm{M}$ cycloheximide. After 16 hours total cathepsin- $B$ enzyme was assayed in the medium $(A)$ and the cell layer extract $(B)$. Each point is the mean of three separate experiments.$\chi^{2}$ test on means of paired samples showed that graph $A-\bullet-$ and $B-\bullet-$ were paired samples showed that graph $A-\bullet$ - and $B-\bullet$ - were
signifly different, $P=0.012$. Also graph $B-\bullet$ and $B$ - $\mathbf{-}$ were significantly different, $P=0.026$. Graph $A-\bullet-$ and $A-\mathbf{-}$ - were not significantly different.

activity in the media. Cathepsin-B activity in the cell-free conditioned medium was determined using the fluorogenic substrate Z-Arg-Arg-AMC, and the fluorescence was standardised to a known amount of AMC. The activity was $100 \%$ inhibited in the presence of $5 \mu \mathrm{M}$ E64, a specific inhibitor of cysteine endopeptidases with high selectivity towards cathepsin-B.

Figure 2 shows a bar graph of the mean of active and total cathepsin-B enzyme in the chondrocyte conditioned medium (CCM) from the three experimental groups at 4,8 , and 12 weeks of experimental osteoarthritis. At 4 weeks there were no statistically significant differences between the mean active cathepsin-B of the osteoarthritic group compared with the control groups. Total enzyme (constitutively active + latent) was higher in all groups, with no significant differences compared with constitutively active enzyme. At eight weeks post-PM the mean cathepsin-B activity of the osteoarthritis group (constitutively active) was significantly higher than that of $\mathrm{N}$ or $\mathrm{SH}$ groups. The total enzyme activity was also significantly higher in PM group than in controls at this time point. However, at 12 


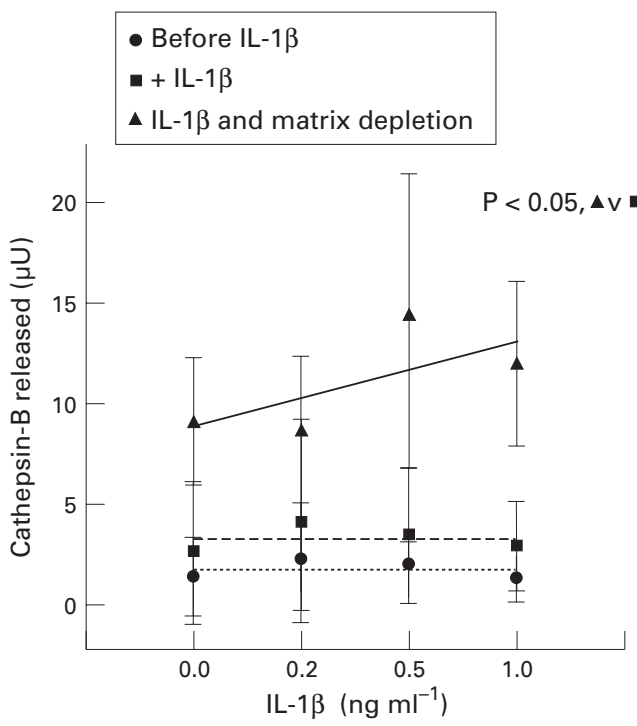

Figure 6 Rabbit articular cartilage explants were cultured for two days, $I L-1 \beta$ was added for a further two days, and culture medium was collected. Explants were subjected to matrix depletion and the cells put in suspension culture for one day; the medium was collected again and total cathepsin- $B$ was assayed in all the media. Each point is the mean of four separate experiments. Regression lines are shown. $\chi^{2}$ test on means of paired samples showed that graphs $-\mathbf{\Delta}-$ and $-\boldsymbol{\square}$ - were significantly different at $\boldsymbol{P}=$ 0.014 .

weeks post-PM the mean active enzyme was not significantly different in the three groups, nor was total enzyme at this time point.

Figure 3 shows the means of the cathepsin-B activity in conditioned media of the synovium from the three groups. Here it was found that at four weeks after surgery the active cathepsin-B was significantly higher in the osteoarthritis group than in the SH group, but not when compared with the $\mathrm{N}$ group. The total activity was not significantly different between the groups. At eight weeks post-PM the mean constitutive activity obtained in the osteoarthritis group was also significantly higher than in the SH controls, but not when compared with the $\mathrm{N}$ controls; total enzyme was not significantly different. At 12 weeks post-PM there were no statistically significant differences between the means of the three groups.

EFFECT OF IL- $1 \beta$ ON CATHEPSIN-B STEADY STATE MRNA, AND ENZYME UPREGULATION IN CHONDROCYTE CULTURES

IL-1 $\beta$ failed to upregulate cathepsin-B mRNA in monolayer chondrocytes. Figure 4 shows a northern blot of cRNA from rabbit monolayer chondrocytes after treatment of cells with $1 \mathrm{ng}$ $\mathrm{ml}^{-1}$ rhIL-1 $\beta$ for various lengths of time. Steady state cathepsin-B mRNA was not detectably altered by stimulation of cells with IL- $1 \beta$ for up to 16 hours following addition of IL-1 $\beta$. However, in the same cells the steady state stromelysin mRNA was upregulated in response to IL-1 $\beta$ within two hours of addition of IL- 1 to cells, as expected (fig 4). The same result was obtained when cartilage explants were used (not shown). Therefore, the response of chondrocyte cathepsin-B steady state mRNA due to stimulation by IL-1 is not dependent on cell shape or phenotypic modulation of chondrocytes.

IL-1 induced a minimal release of cathepsin-B enzyme from chondrocyte monolayers at relatively high doses (fig 5A). By contrast, the intracellular enzyme was upregulated at low doses and then declined to baseline at high doses (fig 5B). Addition of cycloheximide together with IL-1 resulted in blockade of both cathepsin-B synthesis and its secretion (fig 5B and $5 \mathrm{~A}$, respectively). At 0.2 and $0.5 \mathrm{ng} \mathrm{ml}^{-1}$ IL-1 $\beta$, there was higher cathepsin-B in the cell layer than in the medium $(P<0.05)$. Moreover, there was significantly higher cathepsin-B in the cells without cycloheximide than with cycloheximide in the same dose range. Enzyme activity in the medium was not significantly higher with addition of IL-1 than with IL-1 plus cycloheximide. By $\chi^{2}$ test, taking into consideration the entire dose range, addition of IL-1 $\beta$ to the cells resulted in intracellular enzyme levels that were significantly higher than the secreted enzyme $(P=0.012)$. In the absence of cycloheximide, addition of IL- $1 \beta$ to the cells resulted in production of a higher level of intracellular enzyme than with cycloheximide $\left(P=0.026\right.$, by $\left.\chi^{2}\right)$.

When explants were first treated with an optimal dose of IL-1 $\beta$ and were then subjected to matrix depletion and cultured again, this resulted in the release of appreciable quantities of intracellular cathepsin-B relative to intact explants (fig 6); the effect was not due to cell death as cell viability was not affected. Regression analysis showed that only the matrix depleted chondrocytes had a positively sloped dose-response curve compared with nonmatrix depleted cells after treatment with IL-1 $\beta$ alone or before addition of IL- $1 \beta$ to cultures (fig 6). A $\chi^{2}$ test on the mean of the paired points along the dose-response curves showed that the matrix depleted cells released larger amounts of cathepsin-B enzyme into the medium compared with non-matrix-depleted cells $(P=0.014)$.

\section{Discussion}

Data presented in this paper and in previous studies $^{12}$ clearly show that upregulation of cathepsin-B in chondrocytes alone is not an effective stimulus for secretion of the enzyme into the extracellular matrix. For this to happen a second stimulus needs be present. In this study we showed for the first time that an effective stimulus for secretion of active enzyme is matrix depletion. We noted that cathepsin-B steady state mRNA is not detectably altered in response to rhIL-1; stromelysin mRNA was upregulated in the same cells. However, IL-1 brought about upregulation of the intracellular cathepsin-B protein as previously $\operatorname{shown}^{12}$; this upregulation was blocked with an inhibitor of protein synthesis, cycloheximide. Only minute amounts of enzyme were released into culture medium from monolayers or explants when treated with rhIL-1 $\beta$. Therefore the stimulus for cathepsin-B synthesis within the chondrocyte is not the same stimulus that brings about its release. Explants treated 
with $1 \mathrm{ng} \mathrm{ml}^{-1} \mathrm{IL}-1 \beta$ released significantly larger amounts of sulphated proteoglycan fragments (sGAG) into the medium (not shown) suggesting that cartilage catabolism was initiated. Since there was no significant cathepsin-B activity in the medium, this level of degradation was probably due to the action of matrix metalloproteases.

We showed that when explants were treated with IL-1 $\beta$ and then subjected to matrix depletion with bacterial collagenase, there was release of the intracellular enzyme into the medium. Likewise, exposure of monolayers to IL-1 and subsequent treatment with trypsin to dissociate the cells into suspension led to the release of cathepsin-B into the culture medium (not shown). Also it was shown in fig 2 that matrix depleted chondrocytes from knees with experimental osteoarthritis released substantial quantities of cathepsin-B into the culture medium over a three day period. Although the method used here for depletion of matrix is not physiological in the method of enzymatic treatment or the acuteness of matrix loss, it was chosen for the purpose of illustration. It remains to be seen if depletion of matrix with physiological enzymes such as stromelysin or mammalian collagenase produce the same effect.

The steady state cathepsin-B mRNA in chondrocytes from experimental osteoarthritis was not higher than controls at any time point studied in the course of experimental disease. Chondrocytes from EOA rabbit knees secreted relatively large quantities of cathepsin-B; active and total cathepsin-B secreted by EOA chondrocytes was statistically significantly higher at eight weeks compared with $\mathrm{N}$, and $\mathrm{SH}$ controls. This is significant because in osteoarthritis chondrocytes also undergo matrix depletion when the pericellular matrix of the chondrocyte is degraded by the action of other proteases such as stromelysin and collagenase. In a previous study we showed that at eight weeks post-PM pro-stromelysin and pro-collagenase mRNAs are upregulated in EOA chondrocytes. ${ }^{18}$ Unlike cathepsin-B, matrix metalloproteases are always secreted when upregulated in the cell and are not stored. We postulate that the secretion of matrix metalloproteases and subsequent activation by still largely unknown mechanisms would deplete the immediate pericellular matrix of the chondrocyte; this would then serve as the stimulus for the release of cathepsin-B. In this regard it is postulated that chondrocytes may possess an acidic ( $\mathrm{pH}$ 5-6) environment ${ }^{2021}$ at which both stromelysin and cathepsin-B would be highly active. ${ }^{22}$ Therefore cathepsin-B may be involved in osteoarthritis by coming into play at progressive stages of the disease by augmenting the activity of other matrix degrading proteases such as matrix metalloproteases once it has been released following an initial insult. Of interest also is the ability of cathepsin-B to activate pro-stromelysin. ${ }^{23}$ It is possible that pro-stromelysin is activated in vivo by minute amounts of cathepsin-B secreted by chondrocytes or synovium under physiological conditions during normal cartilage matrix remodelling and turnover.

Several molecular forms of secreted cathepsin-B have been noted in culture medium from normal and tumour derived tissues. In human tissues the proenzyme has a molecular mass of approximately $42-46 \mathrm{kDa}$ which, upon activation, produces a molecular species of $\mathrm{Mr} 31-32$ and sometimes $38 \mathrm{kDa}^{2425}$ In a previous study, the rabbit chondrocyte cathepsin-B was characterised as having a molecular size of $\mathrm{Mr} 34 \mathrm{kDa},{ }^{26}$ and was found to be much more resistant to inactivation at neutral and alkaline $\mathrm{pH}$ than the liver derived enzyme. We have not investigated the molecular forms in the culture media of rabbit chondrocytes and synovium in this study; they are, however, likely to correspond to the $34 \mathrm{kDa}$ form described previously. ${ }^{26}$ It is also noteworthy that the chondrocyte derived enzyme is resistant to denaturation at neutral $\mathrm{pH}$; therefore the activities measured in our study are likely to represent the actual activity secreted into the medium.

It should be noted that the stimulus for upregulation and secretion of cathepsin-B described here is not phenotypic modulation or fibroblastic metaplasia, as has been expounded previously for serially subcultured chondrocytes. ${ }^{26}$ Here we found that intact rabbit cartilage explants grown ex vivo contain cathepsin-B that is upregulated in response to IL-1 and is released in significant amounts only after the matrix is subjected to enzymatic depletion. We studied the phenotypic stability of rabbit monolayer chondrocytes cultured as described here without subculturing, and that of chondrocytes maintained for three days in serum-free suspension culture. Both types of cultured cells transcribed mRNA for type II collagen $\alpha 1$ chains (Col $\alpha 1$ (II)) as did freshly isolated cells. Cells cultured in suspension for three days in serum-free medium contained equivalent amounts of $\mathrm{Col} \alpha 1$ (II) mRNA as freshly isolated cells (data not shown).

In the rabbit experimental osteoarthritis model used here, early degenerative changes seen at four weeks after partial meniscectomy are mostly hypertrophic cartilage and a few cartilage erosions. Synovial tissue appears to be involved mainly in the early phase of the disease with involvement of cartilage at later stages. The contribution of the synovium to the enzyme pool within the joint is likely to be a contributory factor in cartilage degradation since the superficial layer of cartilage is the first site of action for degradative enzymes, as evidenced from loss of safranin-O staining and superficial erosions seen in early experimental osteoarthritis. ${ }^{15}$ Synovial cathepsin-B was also found to be secreted in culture but this tissue did not need matrix depletion, presumably because the cells are fundamentally different in the required stimulus for cathepsin-B secretion. Relative to this, spontaneous release of cathepsin-B from synovial cells has been described. ${ }^{13}$ In agreement with the overall synovial and cartilage pathology and the temporal sequence of events seen in this model, synovial cathepsin-B upregulation 
appeared first at four weeks. The mean active cathepsin-B level was significantly increased relative to the $\mathrm{SH}$ controls but not compared with $\mathrm{N}$ controls. This may be due to differences in the activation of pro-enzyme in $\mathrm{N}$ versus $\mathrm{SH}$ conditioned media. At eight weeks post-PM, there was a significant increase in active SCM enzyme in the osteoarthritic group compared with the $\mathrm{SH}$ group; total enzyme was also increased but did not reach statistical significance $(P=0.07)$. It should be noted that although $\mathrm{SH}$ controls do not show any cartilage or synovial pathology and are macroscopically normal in appearance, the cells are in a slightly activated state because of the effect of sham surgery. We have observed this with other indices of disease activity including matrix metalloproteases and cytokines. ${ }^{18}$ This activation maybe related to the release of cytokines/growth factors from soft tissues following the surgical procedure.

In summary, we shown that cathepsin-B is upregulated in chondrocytes and synovial tissue in experimental osteoarthritis at different time points representing disease progression. The synovium appears to be the first source of the enzyme, with cartilage becoming involved as the disease progresses. The upregulation of cathepsin-B is probably cytokine driven, but the release of enzyme from chondrocytes is dependent on environmental factors; this is likely to be depletion of the chondrocyte pericellular matrix. In this regard it is interesting that when a chondrocyte is about to undergo cell division, as seen in the characteristic cloning of chondrocytes in clinical and experimental osteoarthritis, the pericellular matrix of the chondrocyte must be depleted first to allow a chondrocyte to divide. It is possible that cathepsin-B is involved in normal cell division within the matrix and that the pathological state in osteoarthritis represents an uncontrolled lysis of cartilage due to massive release of cathepsin-B from chondrocytes brought about by depletion of the pericellular environment by matrix metalloproteases. These interesting possibilities remain to be explored experimentally.

This study was supported by USPHS AR30134 and AR 20618 , and a research grant from the Procter and Gamble Pharmaceutical Company. We thank Dr Paul Jones of the Department of Biostatistics and Epidemiology of Case Western Reserve University for expert advise with the statistical analyses.

1 Dean DD, Martel-Pelletier J, Pelletier J-P, Howell DS, Woessner JJF. Evidence for metalloproteinase and metalloproteinase inhibitor (TIMP) imbalance in human osteoarthritic cartilage. J Clin Invest 1989;84:678-85.

2 Fosang AJ, Neame PJ, Last K, Hardingham T, Murphy G. The interglobular domain of cartilage aggrecan is cleaved by PUMP, gelatinase and cathepsin B. J Biol Chem 1992; 267:19470-4.

3 Buck MR, Karustis DJ, Day NA, Honn KV, Sloane BF. Degradation of extracellular matrix proteins by human cathepsin B from normal and tumor tissues. Biochem J 1992;282:273-8.
4 Murphy G, Cockett MI, Stephens PE, Smith BJ, Docherty AJP. Stromelysin is an activator of procollagenase. Biochem J 1987;248:265-8.

5 Maciewicz RA, Wolton SF, Etherington DJ, Duance VC. Susceptibility of the cartilage collagens types II, IX and XI to degradation by the cysteine proteinases, cathepsin B and L. FEBS Lett 1990;269:189-93.

6 Nguyen Q, Liu J, Roughley PJ, Mort JS. Link protein as a monitor in situ of endogenous proteolysis in adult human articular cartilage. Biochem J 1991;278:143-7.

7 Martel-Pelletier J, Cloutier JM, Pelletier J-P. Cathepsin B and cysteine protease inhibitors in human osteoarthritis. J Orthop Res 1990;8:336-44.

8 Baici A, Horler D, Lang A, Merlin C, Kissling R. Cathepsin $B$ in osteoarthritis: zonal variation of enzyme activity in human femoral head. Annals Rheum Dis 1995;54:281-8.

9 Baici A, Lang A, Horler D, Kissling R, Merlin C. Cathepsin $\mathrm{B}$ in osteoarthritis: cytochemical and histochemical analysis of human femoral head cartilage. Ann Rheum Dis 1995;54:289-97.

10 Buttle DJ, Handley CJ, Ilic MZ, Saklatvala J, Murata M, Barrett AJ. Inhibition of cartilage proteoglycan release by a specific inactivator of cathepsin B and inhibitor of matrix metalloproteases. Arthritis Rheum 1993;36:1709-17.

11 Esser RE, Angelo RA, Murphey MD, Watts LM, Thornburg $\mathrm{LP}$, Palmer JT, et al. Cysteine proteinase inhibitors
decrease articular cartilage and bone destruction in chronic inflammatory arthritis. Arthritis Rheum 1994; 37:236-47.

12 Baici A, Lang A. Effect of interleukin-1 on the production of cathepsin B by rabbit articular chondrocytes. FEBS Lett 1990;277:93-6.

13 Huet G, Flipo RM, Colin C, Janin A, Hemon B, Hooghe M, et al. Stimulation of the secretion of latent cysteine proteinase activity by tumor necrosis factor- $\alpha$ and interleukin- 1 . Arthritis Rheum 1993;36:772-80.

14 Buttle DJ, Saklatvala J. Lysosomal cysteine endopeptidases mediate interleukin-1 stimulated cartilage proteoglycan degradation. Biochem J 1992;287:657-61.

15 Moskowitz RW, Davis W, Sammarco J, Martens M, Baker J, Mayer M, et al. Experimentally induced degenerative joint lesions following partial meniscectomy in the rabbit. Arthritis Rheum 1973;16:397-405

16 Chan SJ, Segundo BS, McCormick MB, Steiner DF. Nucleotide and predicted amino acid sequences of cloned human and mouse preprocathepsin B cDNAs. Proc Natl Acad Sci USA 1986;83:7721-5.

17 Sanger F, Nicklen S, Coulson AR. DNA sequencing with chain terminating inhibitors. Proc Natl Acad Sci USA 1977;74:5463-7.

18 Mehraban F, Kuo S-Y, Riera H, Chang C, Moskowitz RW. Prostromelysin and procollagenase genes are differentially up-regulated in chondrocyts from the knees of rabbits with experimental osteoarthritis. Arthritis Rheum 1994; 37:1189-97.

19 Gong Q, Chan SJ, Bajkowski AS, Steiner DF, Frankfater A. Characterization of the cathepsin $\mathrm{B}$ gene and multiple mRNAs in human tissues: evidence for alternative splicing of cathepsin B pre-mRNA. DNA Cell Biol 1993;12:299309.

20 Woessner JF, Gunja-Smith Z. Role of metalloproteinases in human osteoarthritis. J Rheumatol (suppl) 1991;27:99101.

21 Etherington DJ. Proteinases in connective tissue breakdown. Ciba Foundation Symposium 1979;87-103.

22 Wilhelm SM, Shao ZH, Housley TJ, Seperack PK, Baumann AP, Gunja Smith Z, et al. Matrix metalloproteinase-3 (stromelysin-1). Identification as the cartilage acid metalloprotease and effect of $\mathrm{pH}$ on catalytic cartilage acid metalloprotease and effect of pH on catalytic 268:21906-13.

23 Murphy G, Ward R, Gavrilovic J, Atkinson S. Physiological mechanisms of metalloproteinase activation. Matrix 1992; suppl 1:224-30.

24 Burnett D, Abrahamson M, Devalia JL, Sapsford RJ, Davies RJ, Buttle DJ. Synthesis and secretion of procathepsin B and cystatin $C$ by human bronchial epithelial cells in vitro: modulation of cathepsin B activity by neutrophil elastase. Arch Biochem Biophys 1995;317:305-10.

25 Werle B, Ebert W, Klein W, Spiess E. Cathepsin B in tumors, normal tissue and isolated cells from the human lung. Anticancer Res 1994;14:1169-76.

26 Baici A, Lang A, Horler D, Knopfel M. Cathepsin B as a marker of the dedifferentiated chondrocyte phenotype. Ann Rheum Dis 1988;47:684-91. 